\title{
Return to Play After a Lateral Ligament Ankle Sprain
}

\author{
P. D'Hooghe ${ }^{1} \cdot$ F. Cruz ${ }^{1} \cdot$ K. Alkhelaifi ${ }^{1}$
}

Published online: 6 May 2020

(C) The Author(s) 2020

\begin{abstract}
Purpose of Review The purpose of this review is to describe the current evidence on the most common sports-related ankle injuries. Joint anatomy, epidemiology, clinical findings, diagnostic approach, and treatment are presented with a specific focus on the available evidence towards return to play.

Recent Findings Recent findings show that ankle sprain is the most common injury in the world of sports. Bony fractures, cartilage defects, and syndesmotic lesions are frequently seen in association with the more severe type of ankle sprains.

Summary In summary, the majority of the athletes' ankle sprains are managed conservatively with excellent outcomes and full return to their pre-injury level of play. However, it is essential to differentiate the single ligament sprain from a more complex injury to the ankle joint. The evidence-based treatment and rehabilitation programmes are associated with a better prognosis and a faster time to return to sport participation.
\end{abstract}

Keywords Ankle injuries $\cdot$ Ligament injuries $\cdot$ Rehabilitation $\cdot$ Return to play

\section{Introduction}

Contact sports are associated with high overall injury rates, both at the professional and amateur level. End-stage ranges in mobility, speed, and directional changes are known to increase the number of injuries [1]. For the ankle, the most common condition in this regard is the so-called "ankle sprain."

The incidence of ankle sprains has been reported to be between 0.324 and 9 per $1000 \mathrm{~h}$ of activity [1,2]. This variability can be related to differences in injury nomenclature and non-homogenous studied populations. Higher ankle sprain rates have been reported predominantly in older athletes, dominant leg, during official games and occurring at the end of each half of a football match [3]. Approximately $60 \%$ of all ankle sprains in athletes arise as a result of direct trauma or due to contact $[4,5]$. The overall ankle re-sprain rate in contact sports is reported to be between 4 and 29\% [3-5].

This article is part of the Topical Collection on Management of Ankle Instability

P. D'Hooghe

Pieter.DHooghe@aspetar.com

1 Department of Orthopaedic Surgery, Aspetar Sports Medicine and Orthopedic Surgery Hospital, Sports City Street 1, Aspire Zone, P.O. Box: 29222, Doha, Qatar
The ankle sprain used to be the most common injury type in professional football players representing $10 \%$ to $36 \%$ of all injuries $[6,7]$, but recent studies proclaim a lower ankle injury rate, representing 10 to $15 \%$ of all injuries $[7,8]$. Potential reasons for this declining trend involve successful injury prevention strategies (e.g. balance training and bracing/taping), stricter game rules, and a more detailed reporting culture of specific injury subtypes [5].

Ankle injuries are nowadays the fourth most common injury type in elite football, and they are preceded by the knee, the thigh, and the lower leg $[7,8]$. Moreover, a recent longterm ankle injury study documented an injury rate of $1 / 1000 \mathrm{~h}$ [5]. This means practically that a professional 25 -player team can expect approximately 7 ankle injuries per season. In terms of overall mean time loss, this represents an average of 16-24 calendar days for every ankle sprain $[1,3,5,9]$. In case of a more severe ankle sprain, this mean number rises to approximately 28 days of absence. In elite football, the ankle sprain represents 10 to $17 \%$ of all related ankle injuries $[5,8,9]$.

\section{Joint Anatomy}

The ankle joint can be mechanically seen as a fork, in which the tibia and both malleoli form a mortise to accommodate the talar bone. As a hinge joint, there is a single axis of movement 
that allows for dorsal flexion $\left(50^{\circ}\right)$ and plantar flexion $\left(20^{\circ}\right)$. The talar bone has a narrower superior surface posteriorly that leads to a looser fit when the fork is moving into plantar flexion. The function of the ankle ligaments is to provide the ankle with the necessary postural stability. The reduced intrinsic intra-articular stability during plantar flexion could explain why most ligamentous injuries are seen in plantar flexion [10]. Inferior to the talocrural joint, the subtalar joint is formed between the inferior surface of the talus and the superior surface of the calcaneus. This subtalar joint provides $35^{\circ}$ of inversion and $15^{\circ}$ of eversion.

The fibula and tibia also articulate distally, creating an inferior tibiofibular joint (supported by the syndesmotic ligaments), and they form the inferior or distal tibiofibular joint (named as "distal tibiofibular syndesmosis"-Fig. 1). It is the syndesmotic joint that allows the tibia-fibula complex to adapt as a whole to the varying width of the upper articular surface of the talar bone, and this minimal movement is vital for enabling normal walking and running. Both passive and dynamic factors provide stability to the ankle joint. Passive stabilization relies on the morphology of the articular surfaces, the articular capsule, surrounding ligamentous complexes, and the retinacula. Dynamic balance is provided by muscle activation mainly.

\section{Lateral Ligament Complex Injury}

Ankle sprains account for 67 to $72 \%$ of all football playerrelated injuries to the ankle $[3,5,9,11,12]$. The ankle joint sprain rate in amateur and professional football players is 2.16 and 0.17 per $1000 \mathrm{~h}$ of exposure, respectively [5, 11]. Most ankle injuries in footballers are caused by player's contact, direct trauma, mostly during tackling (54\%) [9]. Foul play is involved in $40 \%$ of the match-related ankle injuries [5].

Previous sprain injury at the ankle increases 2 to 5 times the chance to have a recurrent ankle sprain compared with players without previous injury $[11,13,14]$. There are no significant gender differences in the overall incidence rate of ankle sprains $[15,16]$. A mean lay off per ankle sprain in football is reported between 7 and 18 days $[5,9,11]$. A total of 83-
$89 \%$ of the ankle sprains require athletes less than 4 weeks of loss of activities $[5,8,9]$, suggesting that it is the incidence rather than the severity of ankle sprains that makes them problematic [9].

However, after standard treatment for an acute sprain ankle, up to $40 \%$ of the patients in the general population report residual symptoms $[17,18]$. These most common residual symptoms are chronic pain, recurrent instability, and muscular weakness. The reported mean costs per ankle sprain are $€ 360.60 \pm 426.73$ [19], but these are unmistakably higher in elite sports.

\section{Aetiology}

Injury to the lateral ligamentous complex represents $70-91 \%$ of all ankle sprains in elite football players $[9,11,20,21]$. This can be partially explained by the relative weakness of the lateral ligaments and the natural tendency for the ankle to go into inversion. The most common mechanism of injury is the inversion of the plantar-flexed foot.

Repetitive video analysis of ankle sprains in football revealed two common mechanisms that put the ankle in this vulnerable position [1]: impact by an opponent on the medial aspect of the lower leg just before or at foot strike, resulting in a laterally directed force causing the footballer to land with the ankle in a vulnerable inverted position [2]. Forced plantar flexion when the injured footballer hits the foot of the opponent when attempting to shoot on the goal or clear the ball [22].

As the anterior talo-fibular ligament (ATFL) is maximally stretched during inversion of the plantar-flexed foot and as it has the lowest tolerance to loads (approximately $150 \mathrm{~N} \mathrm{[23,}$ 24]), the ATFL is the first and often the only ligament injured. As a result, the ATFL is the most frequently injured ligament of the ankle $(90-95 \%[9,11])$. When the mechanism of injury continues around the lateral aspect of the ankle, rupture of the Anterior Talo-Fibular Ligament (ATFL) can cascade to the Calcaneo-Fibular Ligament (CFL), and finally (less frequently) to the PTFL (Fig. 2). It was reported in an MRI study that $41 \%$ of the patients with an ankle inversion trauma have injured both the ATFL and CFL, whereas only $5 \%$ had damaged the PTFL
Fig. 1 The syndesmotic ankle ligaments in 3 views presented
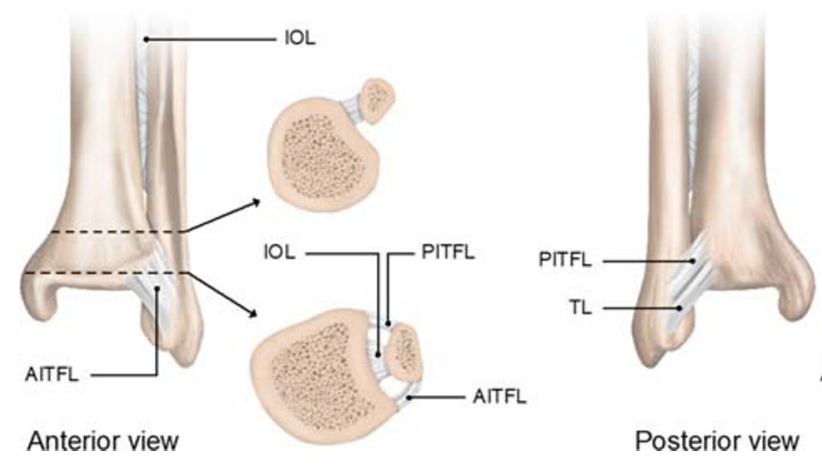

Posterior view

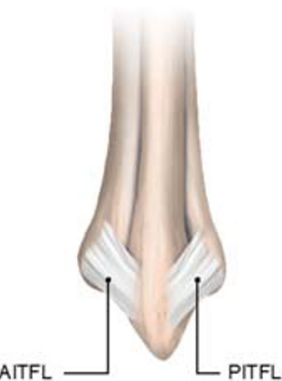

Lateral view 
Fig. 2 Antero-posterior and lateral view to the lateral ankle joint ligaments
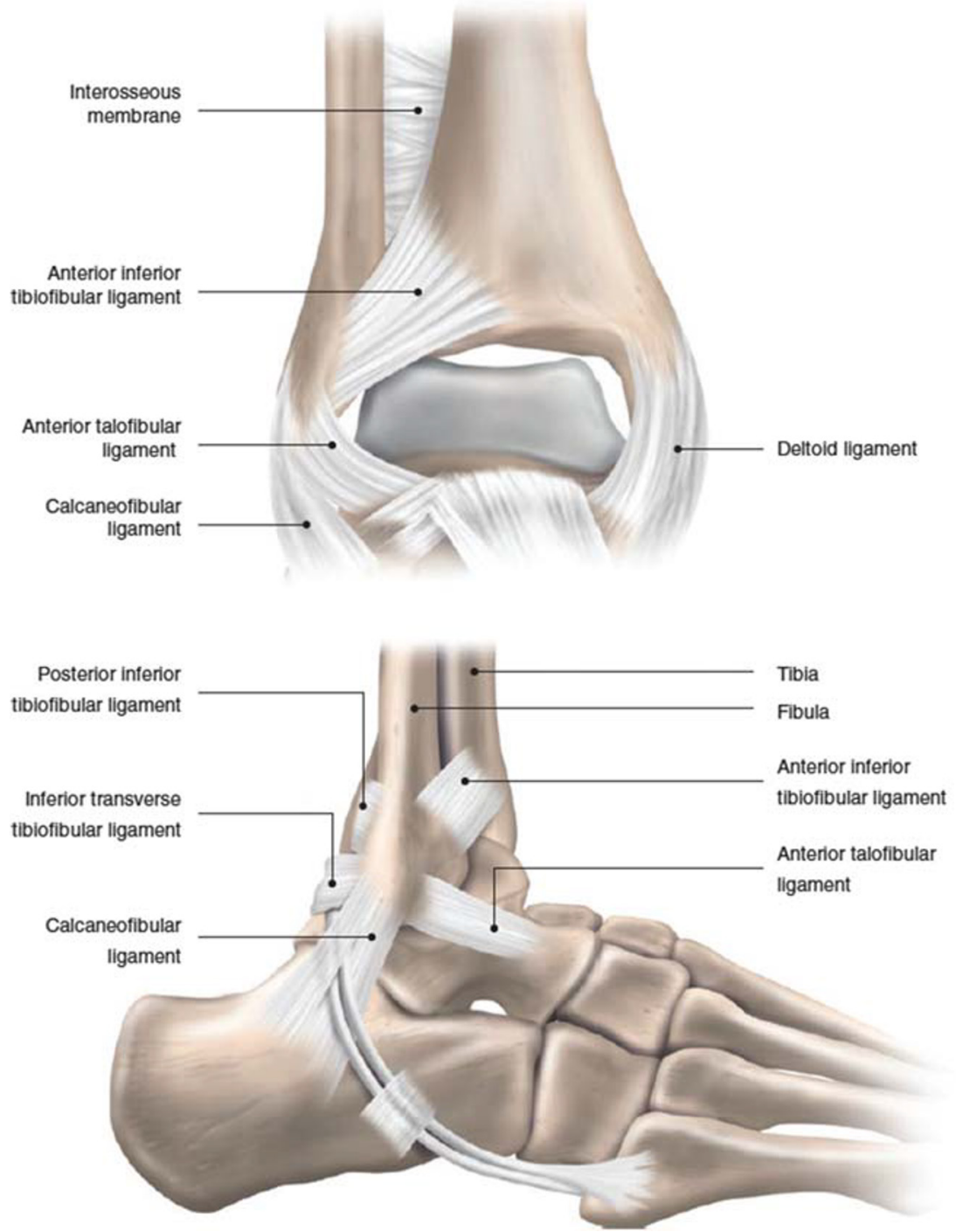

[25]. Associated injuries include bony fractures, osteochondral lesions, and damage to both the peroneus tendon and nerve.

\section{Clinical Findings}

Patient history taking and video analysis are found very useful in studying the injured ligaments. It is essential to distinguish a simple distortion from a lateral ligament rupture since adequate treatment is associated with a better prognosis and time to return to play $[26,27]$. Athletes together with recreants typically report a sudden twisting of the ankle joint, smaller ability to bear weight, and they usually can identify the palpatory painful spot. Patients with lateral ligamentous ruptures report more immediate joint oedema and are more frequently prone to stop their activities [28]. An audible snap or crack sound may accompany ligament sprains. Bony structures and all ligamentous entities around the ankle joint should be palpated for tenderness, including the fibular bone and the base of the fifth metatarsal on the lateral side of the foot. If there is no pain upon palpation over the ATFL, there is probably no lateral ligament rupture apparent $[26,27]$.

Note that approximately $40 \%$ of the athletes with a lateral ligament rupture present with pain upon palpation over the medial malleolus, whereas $60 \%$ report tenderness upon palpation over the AITFL (without rupture of this ligament), probably due to a tear of the anterior capsule [28]. For the physical examination, the anterior drawer test evaluates the ATFL instability, whereas the talar tilt test aims at identifying CFL (calcaneus fibular ligament) instability. If an ecchymosis is present, accompanied by palpatory pain or a positive stress test (or both), it is most likely that a partial lateral ligamentous rupture exists [26, 27].

However, especially in the acute phase, manual stress tests are less reliable due to inhibiting swelling and pain. Therefore, delayed physical examination (after 4-5 days post-injury) of the ankle joint is more reliable and therefore considered the golden standard for diagnosing acute injury to the lateral 
ligament complex of the ankle. Diagnosing an acute lateral ligament rupture during a delayed physical examination has a sensitivity of $96 \%$, with a specificity of $84 \%$ [26, 27].

\section{Diagnosis}

The Ottawa ankle rules help to determine if X-rays are indicated in the assessment of an acute ankle sprain. These Ottawa rules are an instrument to rule out suspected fractures over the ankle after ankle sprain and have a sensitivity close to $100 \%$. Stress radiographs often are not indicated in the routine diagnosis of lateral ligament sprain, as they are challenging to perform and have a relatively low impact on the treatment protocol.

Ultrasound (US) and magnetic resonance scan (MRI) can be useful in the diagnosis of associated injuries (bone, chondral, or tendon). The US has demonstrated to be an accurate tool for investigation of ligament sprains but may be difficult to interpret on retrospective review by other physicians. The sensitivity and specificity of US for a lateral ligament injury is $92 \%$ and $64 \%$, respectively [27]. When US is performed after an inconclusive delayed physical examination, the sensitivity increases to $100 \%$ and specificity to $72 \%$ [27]. A recent study of ATFL injury comparing ultrasonography in the emergency room with MRI images found no overall differences in diagnostic accuracy [29].

MRI is a reliable tool in the diagnosis of injury to the lateral ligamentous ankle complex and associated injuries (Fig. 3), including tendinous and syndesmotic trauma, osteochondral lesions, and occult fractures. The sensitivity and specificity of MRI for ATFL injuries are $92-100 \%$ and $100 \%$, respectively $[30,31]$. In comparison with arthroscopy, MRI images correctly located the injured portion of the ATFL in 93\%, whereas US was able to identify in 63\% [31]. Overall, MRI is the imaging modality of choice for lateral ligamentous injuries over the ankle in elite sports.

\section{Treatment}

A grading system has been created to aid in guiding the treatment of lateral ligament injuries $[32,33]$. This system incorporates anatomical injury with clinical symptoms and is only reliable with delayed physical assessment.

Grade I (mild) injuries include ligament fibre stretch without macroscopic rupture. There is clinically minor swelling and palpatory tenderness, hardly any functional loss, and no increased instability.

Grade II (moderate) injuries include partial ligament tear with moderate pain, swelling and palpatory tenderness, mild to moderate instability, and moderate functional disability.

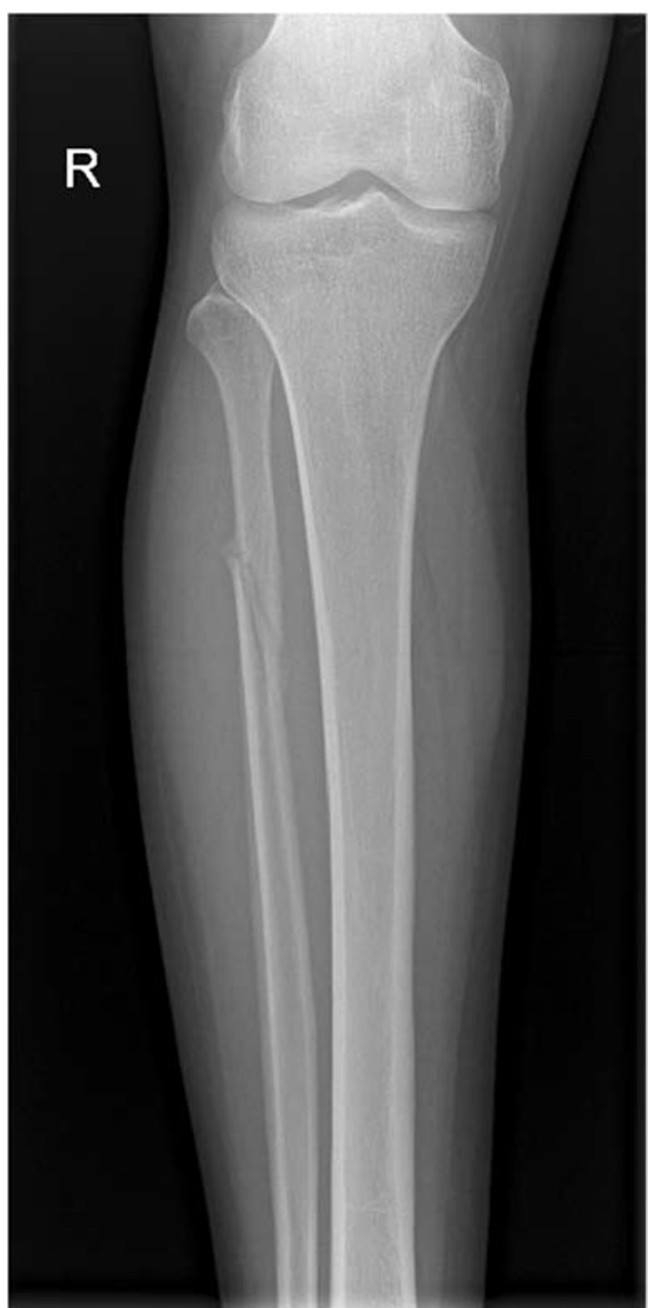

Fig. 3 Maisonneuve fracture after an external rotational injury to the ankle

In grade III (severe) injuries, a complete tear of the ligament and joint capsule rupture is present combined with severe bruising, swelling, and pain. There is a significant loss of function and an increased instability. The athlete is unable to bear weight and walk normal. In clinical treatment practice, only the difference between a simple sprain (grade I) and real instability (grade III) is relevant.

Although there exists a substantial body of research on ankle injuries, controversy remains concerning the best treatment for acute lateral ligament injuries, especially for grade III injuries in elite athletes. However, non-operative measures can be prescribed for the majority of acute grades I-III lateral ligament sprains with good to excellent outcome [34].

Treatment of lateral ligament tears is based on the 3 phases of biological ligament healing: inflammatory phase, proliferation phase (6 to 12 weeks post-trauma), and remodelling or maturation phase (until 1-year post-injury).

Initially, the treatment during the inflammatory phase is aimed towards avoiding swelling and ongoing injury. This is achieved with the POLICE (protect, optimal loading, ice, 
compression, elevation) protocol for the first 4-5 days [35]. Oral or topical use of nonsteroidal anti-inflammatory drugs (NSAID) decreases pain without increase in adverse events [36]. Manual mobilization therapy of the ankle joint has minimal benefits and should be discouraged [37]. No effect was found from therapies like low-intensity ultrasound, laser, or electrotherapy [37]. Long-term immobilization ( $>2$ weeks) leads to poorer outcomes than functional treatment $[34,38]$. However, a short time of immobilization (maximum 10 days) in a cast below the knee or removable boot can be advantageous for severe lateral ligamentous tears (faster recovery compared with compression bandage) $[34,39]$.

In the proliferation phase, fibroblasts proliferate and new collagen is formed. Controlled stress on the damaged ligament will promote proper collagen fibres orientation, whereas protection of ankle inversion is essential to prevent the excess formation of weaker type III collagen.

Hereby, the prescription of an external ankle brace is advocated. Treatment with elastic bandages results in fewer complications versus taping but is associated with a slower return to sport and remaining instability versus a semi-rigid ankle brace [40]. Although a recent study reported no differences in outcome 6 months after treatment with tape, semi-rigid brace, or lace-up brace [41], a significant number of studies report superior results from protection with a brace [34, 42]. The use of a (semi-rigid) brace in the proliferation phase is preferred since it is more cost-effective and leads to fewer skin complications. Exercise therapy combined with progressive weight bearing is also an essential stage in the functional treatment of acute lateral ligamentous sprain [43]. Early active range of motion (ROM) exercises are subsequently followed by strengthening exercises, proprioceptive training, and functional exercises. Activities in this final phase should progressively simulate the physical demands of the respective sport modality during practise sessions and competition that includes jumping, turning, and twisting. Supervision by a multidisciplinary team and knowledgeable sport medicine staff is necessary and can be considered for all athletes, although unsupervised, home-based training was also reported to be effective [44]. Ideal rehabilitation programme schedules for acute lateral ligamentous injuries, based on current best evidence, have been described [45-47]. However, there is still a lack of research to design specific rehabilitation protocols for returning athletes to sports competition.

The treatment of grade III lateral ligament ankle injury remains controversial. Most reviews comparing surgery versus conservative treatment for acute lateral ankle ligament injuries failed to demonstrate a superior treatment approach $[34,42]$. Therefore, functional treatment is preferred over surgery in most of the cases [34, 42]. However, surgical treatment may be beneficial on an individual basis in elite athletes [48]. The advantage of surgical repair is significantly less objective instability when compared with non-operative treatment [43].
Since increased instability is predictive for future ankle sprains [49] and return to sports is not delayed after surgical treatment [48], acute surgical repair should be considered in professional football players $[28,34,48]$.

Timing in the season, expectations of the athlete, sportsspecific ankle load, individual history, stage of athlete career, time from trauma to diagnosis, combined injury, and access to expert medical imaging and treatment are all features to be assessed when considering surgery [28]. When indicating a surgical repair in an acute injury, a direct anatomical reconstruction of the ruptured lateral ligaments in a high-volume centre by an experienced ankle sports surgeon is recommended $[28,48]$. The rehabilitation regime after direct anatomic reconstruction, as described in a recent evidence-based guideline [50], is lower-leg cast for 1 or 2 weeks, followed by 2 4 weeks in a walking boot and an active rehabilitation protocol with the use of an ankle support.

\section{Return to Play}

After a lateral ligamentous injury, it is difficult to predict precisely when the athlete can return to sports (RTS). Furthermore, residual disability of ankle joint sprains is often caused by an inadequate rehabilitation programme and early RTS [46]. The current literature lacks formal criteria to assist in the decision to RTS of athletes with a lateral ligament injury. When analyzing the ability of an athlete to return to sports activities, all functional limitations as a result of the damage have to be restored, cardiovascular fitness should be equal to or greater than pre-injury status, and there should be no apprehension from the athlete or other members of the rehabilitation team concerning the health safety of the athlete. The RTS process itself will often be progressive as well, and objective data are required to assess the ability of the athlete to progress to the next rehabilitation phase. Although self-reported ankle scoring systems (e.g. FAOS [51]) are not validated for RTS decisions, they can be useful to evaluate the effectiveness of the rehabilitation protocol. Moreover, the use of functional performance tests is considered helpful to assess the ability to perform sport-specific athletic skills again [45].

Tests can progress from relatively simple tasks (like the single-legged balance test [52]) to more complex tasks (such as the Star Excursion Balance Test [53], the Y-balance test [54], and the agility T-test [55]). The outcome of these tests should be evaluated throughout the rehabilitation process, thereby quantifying progress and comparison against preinjury level and the contralateral side. As several functional tests are predictive of ankle injuries in uninjured athletes [55-58], the use of these tests in the RTS decision of athletes with lateral ligamentous ankle injury should be validated. A minimal score on the functional test for RTS, e.g. $90 \%$ of preinjury or contralateral side, has been advised [44] but warrants 
further research [45]. In the case of RTS, the rehabilitation programme should never be stopped abruptly, as deficits can erroneously be overlooked during the return to play evaluation. Moreover, specific gaps may only be present after the athlete has been thoroughly fatigued. Continuing sportspecific rehabilitation will help to minimize this risk.

The time needed to RTS in lateral ligamentous ankle sprains depends on several factors, including the severity of the injury, the ability of the athlete, and the rehabilitation features available. The reported RTS in amateur and professional football players has been between 7 and $15 \pm 19$ days, respectively $[5,11]$. There was no documentation on the gradation of the injuries. A case series of professional athletes who underwent surgical ligament repair reported a median RTS of 77 days for isolated lateral ligamentous injuries and 105 days for those with concomitant injuries [50].

The most critical risk factor for an ankle sprain is the history of a previous ankle sprain due to a reduced mechanical stability and reduced proprioceptive ability. There is evidence that neuromuscular training, especially balance training (e.g. wobble board), is useful in the prevention of recurrent ankle sprains. This type of therapy can also be effectively performed at home [44]. It is controversial if neuromuscular training is beneficial in healthy ankles in preventing the first presentation of a sprain [28]. There is a consensus that external ankle brace reduces the risk of recurrent ankle injury in previously injured athletes [40] by approximately 70\% [59]. These results were reproduced in football players $[7,60]$. It is unclear whether an external brace is more effective than taping [48], since both have their advantages and disadvantages. The taping technique can lead to skin lesions and loses $40-50 \%$ of its effectiveness after $15 \mathrm{~min}$ of intensified exercise [61]. However, some athletes tend to dislike braces because they do not fit well in the usual football shoes. Braces are re-usable and re-adjustable, and minimal expertise is required for correct installation. Contrary to popular belief, external ankle support does not impede speed, agility, and kicking accuracy in football players $[62,63]$. A combination of both treatment modalities can therefore be considered.

\section{Fact Box}

- Delayed physical examination (4-5 days) of the lateral ankle ligament complex gives better results than the one that is done within $48 \mathrm{~h}$.

- The majority of acute lateral ligament injuries of the ankle can be treated conservatively with an adequate rehabilitation protocol.

- Surgical treatment can be considered in high-level athletes with acute grade III injuries.
- Surgery provides a lower incidence of chronic ankle instability versus conservative treatment.

- RTS criteria should include functional performance tests (e.g. $90 \%$ score)

\section{Conclusions}

This review describes the current evidence on the most common sports-related ankle injuries with a specific focus on the available evidence towards return to play. The majority of the athletes' ankle sprains are managed conservatively with excellent outcomes and full return to their pre-injury level of play. More severe - grade III-lateral ligament ankle injury remains controversial. Acute surgical repair should be considered in professional football players, especially when increased instability is present. RTP management is a multifactorial and interdisciplinary process. Individualized assessment should focus on patient profile, injury type, and sports modality.

Funding Information Open Access funding provided by the Qatar National Library.

\section{Compliance with Ethical Standards}

Conflict of Interest Pieter D'Hooghe, Flavio Cruz, and Khalid Al Khelaifi declare that they have no conflict of interest.

Human and Animal Rights and Informed Consent This article does not contain any studies with human or animal subjects performed by any of the authors.

Open Access This article is licensed under a Creative Commons Attribution 4.0 International License, which permits use, sharing, adaptation, distribution and reproduction in any medium or format, as long as you give appropriate credit to the original author(s) and the source, provide a link to the Creative Commons licence, and indicate if changes were made. The images or other third party material in this article are included in the article's Creative Commons licence, unless indicated otherwise in a credit line to the material. If material is not included in the article's Creative Commons licence and your intended use is not permitted by statutory regulation or exceeds the permitted use, you will need to obtain permission directly from the copyright holder. To view a copy of this licence, visit http://creativecommons.org/licenses/by/4.0/.

\section{References}

1. Cloke DJ, Ansell P, Avery P, Deehan D. Ankle injuries in football academies: a three-centre prospective study. Br J Sports Med. 2011;45(9):702-8.

2. Giza E, Fuller C, Junge A, Dvorak J. Mechanisms of foot and ankle injuries in soccer. Am J Sports Med. 2003;31(4):550-4. 
3. Cloke DJ, Spencer S, Hodson A, Deehan D. The epidemiology of ankle injuries occurring in English football association academies. Br J Sports Med. 2009;43(14):1119-25.

4. Jain N, Murray D, Kemp S, Calder J. Frequency and trends in foot and ankle injuries within an English premier league football club using a new impact factor of injury to identify a focus for injury prevention. Foot Ankle Surg. 2014;20(4):237-40.

5. Waldén M, Hägglund M, Ekstrand J. Time-trends and circumstances surrounding ankle injuries in men's professional football: an 11-year follow-up of the UEFA champions league injury study. Br J Sports Med. 2013;47(12):748-53.

6. Anderson RL, Engebretsen L, Kennedy N, LaPrade R, Wegner AM, Giza E (2014) Epidemiology and mechanisms of ankle pathology in football. In: The aAnkle in Football. Springer-Verlag, Paris, p 31-59.

7. Stubbe JH, van Beijsterveldt AM, van der Knaap S, Stege J, Verhagen EA, van Mechelen W, et al. Injuries in professional male soccer players in the Netherlands: a prospective cohort study. J Athl Train. 2015;50(2):211-6.

8. Ekstrand J, Hägglund M, Waldén M. Injury incidence and injury patterns in professional football: the UEFA injury study. Br $\mathrm{J}$ Sports. 2011;45(7):553-8.

9. Woods C, Hawkins R, Hulse M, Hodson A. The Football Association Medical Research Programme: an audit of injuries in professional football: an analysis of ankle sprains. Br J Sports Med. 2003;37(3):233-2388

10. Giza E (2006) Chronic footballer's ankle. In: Football traumatology, Springer Milan, pp 333-351.

11. Kofotolis ND, Kellis E, Vlachopoulos SP. Ankle sprain injuries and risk factors in amateur soccer players during two year period. Am J Sports Med. 2007;35(3):458-66.

12. Price RJ, Hawkins RD, Hulse MA, Hodson A. The Football Association medical research programme: an audit of injuries in academy youth football. Br J Sports Med. 2004;38(4):466-71.

13. Arnason A, Sigurdsson SB, Gudmundsson A, Holme I, Engebretsen L, Bahr R. Risk factors for injuries in football. Am J Sports Med. 2000;32(1 Suppl):5S-16S.

14. Vereijken AJ (2012) Risk factors for ankle sprain injury in male amateur soccer players: a prospective cohort study. http://dspace. library.uu.nl/handle/1874/252485.

15. Emery CA, Meeuwisse WH. The effectiveness of a neuromuscular prevention strategy to reduce injuries in youth soccer: a clusterrandomised controlled trial. Br J Sports Med. 2010;44(8):555-62.

16. Waterman BR, Owens BD, Davey S, Zacchilli MA, Belmont PJ Jr. The epidemiology of ankle sprains in the United States. J Bone Joint Surg Am. 2010;92(13):2279-84.

17. Ferran NA, Maffulli N. Epidemiology of sprains of the lateral ankle ligament complex. Foot Ankle Clin. 2006;11(3):659-62.

18. van Rijn RM, van Os AG, Bernsen RMD, Luijsterburg PA, Koes BW, Bierma-Zeinstra SMA. What is the clinical course of acute ankle sprains? A systematic literature review. Am J Med. 2008;121:324-31.

19. Verhagen EA, van Tulder M, van der Beek AJ, Bouter LM, van Mechelen W. An economic evaluation of a proprioceptive balance board training programme for the prevention of ankle sprains in volleyball. Br J Sports Med. 2005;2:111-5.

20. Ekstrand J, Gillquist J. Soccer injuries and their mechanisms: a prospective study. Med Sci Sports Exerc. 1983;15(3):267-70.

21. Hawkins RD, Hulse MA, Wilkinson C, Hodson A, Gibson M. The association football medical research programme: an audit of injuries in professional football. Br J Sports Med. 2001;35(1):43-7.

22. Andersen TE, Floerenes TW, Arnason A, Bahr R. Video analysis of the mechanisms for ankle injuries in football. Am J Sports Med. 2004;32(1 Suppl):69S-79S.

23. Krips R, de Vries J, van Dijk CN. Ankle instability. Foot Ankle Clin. 2006;11(2):311-29.
24. St Pierre RK, Rosen J, Whitesides TE, Szczukowski M, Fleming LL, Hutton WC. The tensile strength of the anterior talofibular ligament. Foot Ankle. 1983;4(2):83-5.

25. Khor YP, Tan KJ. The anatomic pattern of injuries in acute inversion ankle sprains: a magnetic resonance imaging study. Orthop J Sports Med 2013. 2013;1(7):2325967113517078.

26. Van Dijk CN, Lim LS, Bossuyt PM, Marti RK. Physical examination is sufficient for the diagnosis of sprained ankles. J Bone Joint Surg Br. 1996;78(6):958-62.

27. Van Dijk CN, Mol BW, Lim LS, Marti RK, Bossuyt PM. Diagnosis of ligament rupture of the ankle joint. Physical examination, arthrography, stress radiography, and sonography compared in 160 patients after inversion trauma. Acta Orthop Scand. 1996;67(6):566-70.

28. van den Bekerom MP, Kerkhoffs GM, McCollum GA, Calder JD, van Dijk CN. Management of acute lateral ankle ligament injury in the athlete. Knee Surg Sports Traumatol Arthrosc. 2013;21(6): 1390-5.

29. Gün C, Unlüer EE, Vandenberk N, Karagöz A, Sentürk GO, Oyar O. Bedside ultrasonography by emergency physicians for anterior talofibular ligament injury. J Emerg Trauma Shock. 2013;6(3):1958.

30. Joshy S, Abdulkadir U, Chaganti S, Sullivan B, Hariharan K. Accuracy of MRI scan in the diagnosis of ligamentous and chondral pathology in the ankle. Foot Ankle Surg. 2010;16(2):78-80.

31. Oae K, Takao M, Uchio Y, Ochi M. Evaluation of anterior talofibular ligament injury with stress radiography, ultrasonography, and MR imaging. Skelet Radiol. 2010;39(1):41-7.

32. Hamilton WG. Sprained ankles in ballet dancers. Foot Ankle. 1982;3(2):99-102.

33. Kaikkonen A, Kannus P, Järvinen M. A performance test protocol and scoring scale for the evaluation of ankle injuries. Am J Sports Med. 1994;22(4):462-9.

34. Petersen W, Rembitzki IV, Koppenburg AG, Ellermann A, Liebau C, Brüggemann GP, et al. Treatment of acute ankle ligament injuries: a systematic review. Arch Orthop Trauma Surg. 2013;133(8): $1129-41$.

35. Bleakley CM, Glasgow P, MacAulley DC. PRICE needs updating, should we call the POLICE? Br J Sports Med 2012. 2011;46:220 1. https://doi.org/10.1136/bjsports-2011-090297.

36. van den Bekerom MP, Sjer A, Somford MP, Bulstra GH, Struijs PA, Kerkhoffs GM. Non-steroidal anti-inflammatory drugs (NSAIDs) for treating acute ankle sprains in adults: benefits outweigh adverse events. Knee Surg Sports Traumatol Arthrosc. 2015;23(8):2390-9.

37. Kerkhoffs GM, van den Bekerom M, Elders LA, van Beek PA, Hullegie WA, Bloemers GM, et al. Diagnosis, treatment and prevention of ankle sprains: an evidence-based clinical guideline. Br J Sports Med. 2012;46(12):854-60.

38. Kerkhoffs GM, Rowe BH, Assendelft WJ, Kelly K, Struijs PA, van Dijk CN. Immobilisation and functional treatment for acute lateral ankle ligament injuries in adults. Cochrane Database Syst Rev. 2002;3:CD003762.

39. Lamb SE, Marsh JL, Hutton JL, Nakash R, Cooke MW, Collaborative Ankle Support Trial (CAST Group). Mechanical supports for acute, severe ankle sprain: a pragmatic, multicentre, randomized controlled trial. Lancet. 2009;373(9663):575-81.

40. Kerkhoffs GM, Struijs PA, Marti RK, Assendelft WJ, Blankevoort L, van Dijk CN. Different functional treatment strategies for acute lateral ankle ligament injuries in adults. Cochrane Database Syst Rev. 2002;3:CD002938.

41. van den Bekerom MP, van Kimmenade R, Sierevelt IN, Eggink K, Kerkhoffs GM, van Dijk CN, et al. Randomized comparison of tape versus semi-rigid and versus lace-up ankle support in the treatment of acute lateral ankle ligament injury. Knee Surg Sports Traumatol Arthrosc. 2016;24(4):978-84. 
42. Kemler E, van de Port I, Backx F, van Dijk CN. A systematic review on the treatment of acute ankle sprain: brace versus other functional treatment types. Sports Med. 2011;41(3):185-97.

43. Kerkhoffs GM, Handoll HH, de Bie R, Rowe BH, Struijs PA. Surgical versus conservative treatment for acute injuries of the lateral ligament complex of the ankle in adults. Cochrane Database Syst Rev. 2007;2:CD000380.

44. Hupperets MD, Verhagen EA, van Mechelen W. Effect of unsupervised home based proprioceptive training on recurrences of ankle sprain: randomized controlled trial. BMJ. 2009;339:b2684.

45. Kaminski TW, Hertel J, Amendola N, Docherty CL, Dolan MG, Hopkins JT, et al. National Athletic Trainers' association position statement: conservative management and prevention of ankle sprains in athletes. J Athl Train. 2013;48(4):528-45.

46. Renström PA, Konradsen L. Ankle ligament injuries. Br J Sports Med. 1997;31(1):11-20.

47. Zöch C, Fialka-Moser V, Quittan M. Rehabilitation of ligamentous ankle injuries: a review of recent studies. Br J Sports Med. 2003;37(4):291-5.

48. Kerkhoffs GM, Tol JL. A twist on the athlete's ankle twist: some ankles are more equal than others. Br J Sports Med. 2012;46(12): 835-6.

49. Verhagen EA, Van der Beek AJ, Bouter LM, Bahr RM, Van Mechelen W. A one season prospective cohort study of volleyball injuries. Br J Sports Med. 2004;38(4):477-81.

50. Pearce CJ, Tourné Y, Zellers J, Terrier R, Toschi P, Silbernagel KG, et al. Rehabilitation after anatomical ankle ligament repair or reconstruction. Knee Surg Sports Traumatol Arthrosc. 2016;24(4):1130-9.

51. Roos EM, Brandsson S, Karlsson J. Validation of the foot and ankle outcome score for ankle ligament reconstruction. Foot Ankle Int. 2001;22(10):788-94.

52. Freeman MA, Dean MR, Hanham IW. The etiology and prevention of functional instability of the foot. J Bone Joint Surg Br. 1965;47(4):678-85.

53. Hertel J, Miller SJ, Denegar CR. Intratester and intertester reliability during the star excursion balance test. J Sport Rehabil. 2009;9(2): 104-16.
54. Plisky PJ, Gorman PP, Butler RJ, Kiesel KB, Underwood FB, Elkins B. The reliability of an instrumented device for measuring components of the star excursion balance test. N Am J Sports Phys Ther. 2009;4(2):92-9.

55. Pauole K, Madole K, Garhammer J, Lacourse M, Rozenek R. Reliability and validity of the T-test as a measure of agility, leg power, and leg speed in college aged men and women. J Strength Cond Res. 2000;14(4):443-50.

56. Gonell AC, Romero JA, Soler LM. Relationship between the Ybalance test scores and soft tissue injury incidence in a soccer team. Int J Sports Phys Ther. 2015;10(7):955-66.

57. McGuine TA, Greene JJ, Best T, Leverson G. Balance as a predictor of ankle injuries in high school basketball players. Clin J Sports Med. 2000;10(4):239-44.

58. Plisky PJ, Rauh MJ, Kaminski TW, Underwood FB. Star excursion balance test as a predictor of lower extremity injury in high school basketball players. J Orthop Sports Phys Ther. 2006;36(12):911-9.

59. Dizon JM, Reyes JJ. A systematic review on the effectiveness of external ankle supports in the prevention of inversion ankle sprains among elite and recreational players. J Sci Med Sport. 2010;13(3): 309-17.

60. Sharpe SR, Knapik J, Jones B. Ankle braces effectively reduce recurrence of ankle sprains in female soccer players. J Athl Train. 1997;32(1):21-4.

61. Frankeny JR, Jewett DL, Hanks GA, Sebastianelli WJ. A comparison of ankle-taping methods. Clin J Sports Med. 1993;3(1):20-5.

62. Paris DL. The effects of the Swede-O, New Cross, and McDavid ankle braces and adhesive ankle taping on speed, balance, agility, and vertical jump. J Athl Train. 1992;27(3):253-6.

63. Putnam AR, Bandolin SN, Krabak BJ. Impact of ankle bracing on skill performance in recreational soccer players. PM R. 2012;4(8): 574-9.

Publisher's note Springer Nature remains neutral with regard to jurisdictional claims in published maps and institutional affiliations. 